\title{
EXPERIMENTAL AND SIMULATION STUDIES ON THE VOLTAGE DROP OF ARC IN LOW-VOLTAGE CIRCUIT BREAKER
}

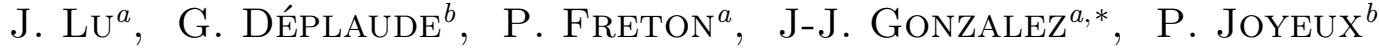 \\ ${ }^{a}$ Laboratory on Plasma and Conversion of Energy, University of Toulouse, CNRS, INPT, UPS., 118 Route de \\ Narbonne, F-31062 Toulouse cedex 9, France \\ ${ }^{b}$ Hager Electro SAS, 132 Bd d'Europe, 67215 Obernai, France \\ * gonzalez@laplace.univ-tlse.fr
}

\begin{abstract}
In low voltage circuit breaker (LVCB) apparatus, a current limitation is performed by increasing the arc voltage. This increase is mainly realized in the splitters plates of the arc chamber by additional drop voltages due to anode and cathode sheaths regions. The consideration of the voltage drops near-electrodes regions is so one of the most important mechanism to improve the description of the arc behavior in LVCB. In this paper, the arc voltage evolution has been studied by experimental and simulation by considering a simple geometry constituted by two rails runner with one or two splitters plates. One magneto hydrodynamic model in three dimensions (3D) was developed to simulate the arc motion and the arc splitting process. In order to compare with the model results, experimental tests have been carried out.
\end{abstract}

Keywords: Low-voltage circuit breaker, voltage drop, splitters, experiment, simulation.

\section{Introduction}

The principle of LVCB consists to create a current limitation by increasing the arc voltage. The increase of the voltage is carried out by various mechanisms: increase of the losses, arc elongation and multiplication of the voltage drops in the splitters. To quantify and better describe these mechanisms, studies are conducted through models and experiments [1-4]. The most theoretical approach studied and applied in LVCB is the one proposed by Lindmayer [2]. This method allows considering an additional drop voltage through the resistivity in the layers. Another approach consists to describe the sheath with a nonequilibrium approach [5]. Nevertheless this method is difficult to implement for a real configuration with several splitters plates. Some authors [6-10] studied numerically the splitting process. Considering a nonlinear permeability of ferromagnetic material's they focus their studies on the influence of eddy currents [9] and on the influence of metal vapors coming from iron splitter plate erosion [10]. In those studies, the cathode voltage was treated by U-J curves and the anode arc root description was determinedwith the LTE-diffusion model of Lowke and Tanaka [11]. Another group has studied the arc phenomena in LVCB by an imaging system applied to a flexible test apparatus [12-15]. The studies were devoted to the influence of vent aperture size on re-ignition and arcing phenomena. They also proposed a modified method to consider the voltage due to the sheath regions. The ability to predict the arc extinction was analyzed and compared [16] with the Lindmayer approach [2]. The most complete study was proposed by Iturregi et al. [17]. They designed a 3D model in LVCB to analyze the arc behavior. Nevertheless incoherencies appear in the comparison between one and two splitters plate: the additional drop voltage due to a second splitter is not present in the total voltage evolution versus time. The method is not clearly described as the authors used directly the @Ansys module which includes the Lindmayer approach. In this paper, the process of arc splitting is analyzed by theoretical and experimental works. Experimental tests have been realized using one and two splitters: high speed camera and electrical measurements. For the model we have implemented the Lindmayer approach and used the same geometries of Iturregi et al. [17].

\section{Numerical method}

\subsection{Hypotheses}

The following assumptions have been used:

(1) The air medium plasma is assumed to be in Local Thermodynamic Equilibrium (LTE).

(2) The plasma is a Newtonian fluid and the flow is laminar.

(3) Vapors from the walls and electrodes are not taken into account.

(4) Radiation is treated using the net emission coefficient method.

\subsection{Equations}

Based on the Fluent software, we have implemented a fluid model to simulate electrical arc behavior in a LVCB. It can be characterized by the macroscopic fluid quantities: velocity, pressure, enthalpy. All these 
quantities are obtained from the Navier-Stokes equations with Lorentz forces and homic heating and completed with Maxwell equations to consider electromagnetism effects. The magnetic field is obtained from the current path in the runners and plasma through the vector potential resolution and a BiotSavart formulation is used at the boundaries. In order to represent the additional voltage drop for the runners and splitters, an effective electrical conductivity is defined [2] based on experimental works $[6,8]$. The voltage evolution versus the current density is plotted in the Figure 1. These characteristics (a-b-c-d) suggested by Lindmayer et al. [2] in air medium with copper runners enable some tuning of the model to adjust the voltage, they represent the current ability to jump on the splitter plate.

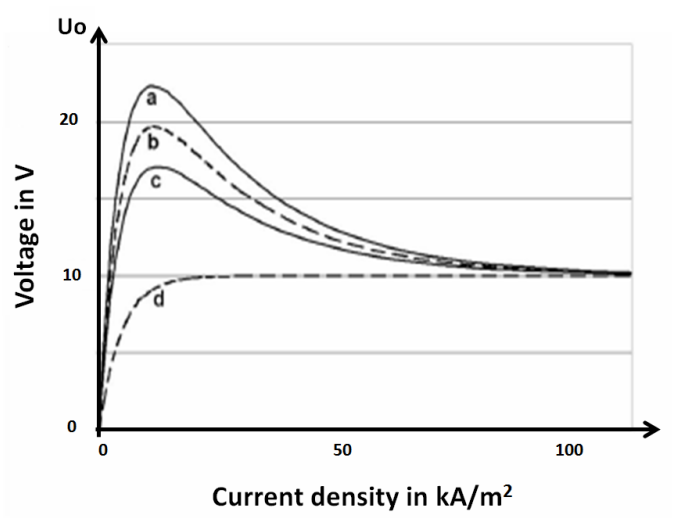

Figure 1. Evolution of voltage drop versus current density [3]

\subsection{Boundary conditions}

"Classical" boundary conditions are used [1]. The magnetic field is calculated from the vector potentials but the BiotSavart formulation is used to close the system resolution.

\subsection{Geometries}

The geometries for the simulation are presented in the Figure 2. The dimensions of the chambers are $40 \times 2.5 \times 11 \mathrm{~mm}(x y z)$ and the rails are $40 \times 2.5 \times$ $1.5 \mathrm{~mm}(x y z)$. It should be noted that the dimension of the splitters plate are not the same: for one splitter: $20 \times 2.5 \times 2 \mathrm{~mm}(x y z)$ and for two splitters: $20 \times$ $2.5 \times 1 \mathrm{~mm}(x y z) . D 1=8 \mathrm{~mm}$ represents the distance between the two runners; $D 2=3 \mathrm{~mm}$ is the distance between runners and splitters, for the third geometry $D 3=2 \mathrm{~mm}$. These dimensions are far from real LVCB but chosen to correspond with Iturregi et al. [17]. A uniform grid is used in the geometry leading to 1.12 million cells.

\section{Simulation results}

We present the main results obtained with the 3D model applying the Lindmayer approach. The arc

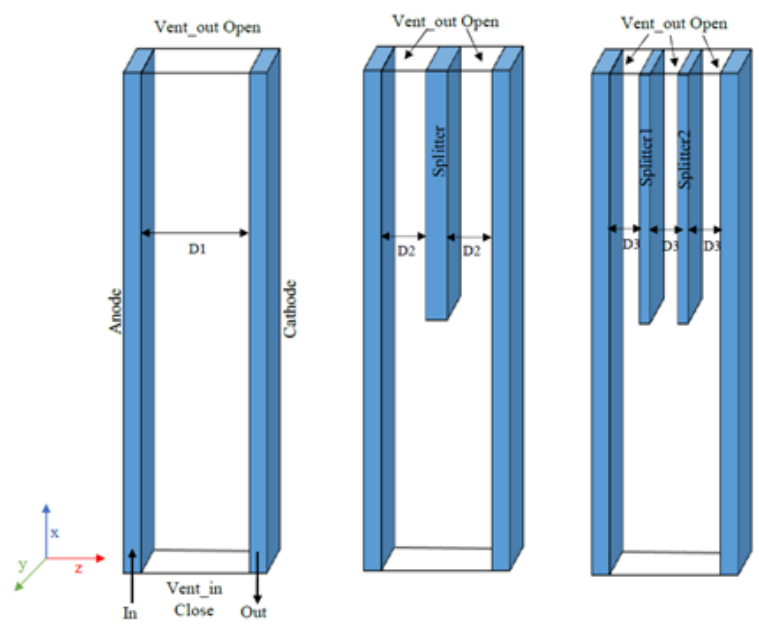

Figure 2. Geometries used for the simulation (From left to right: 0 splitter, 1 splitter and 2 splitters).

splitting behavior is shown through the current densities in Figure 4 for one and two splitter plates. Figure 3 presents the voltage evolution for the two cases. The calculation time step is $10 \mu \mathrm{s}$ and the input current is $50 \mathrm{~A}$ DC. The curve "c" of Figure 1 was chosen to simulate the sheath contribution. There is no symmetry plane in our simulation. In order to reduce the calculation time, the arc is ignited $1.5 \mathrm{~mm}$ front of splitters plates.

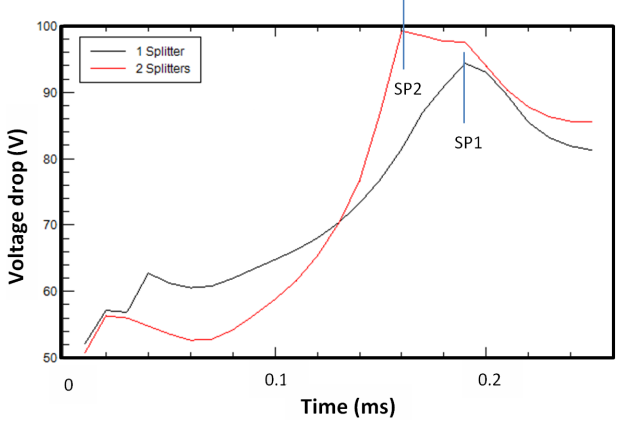

Figure 3. Voltage drop in the simulation with 1 and 2 splitters.

In case of one splitter: At $t=50 \mu \mathrm{s}$ the arc starts to bend and moves toward the splitters. Due to the arc elongation the voltage increases progressively from $61 \mathrm{~V}$ at $t=50 \mu \mathrm{s}$ to its maximum value 94 $\mathrm{V}$ at $t=0.19 \mathrm{~ms}$. Then the voltage decreases. At $t=0.19 \mathrm{~ms}$ the drop voltage is due to the effects of the arc elongation and to the additional voltage sheathes. After time $t=0.19 \mathrm{~ms}$, the current flows progressively into the splitter leading to a diminution of the current path and of the total voltage. The current path goes into the splitter totally at $t=0.21 \mathrm{~ms}$. At $t=0.25 \mathrm{~ms}$ there is only one path of current and the voltage drop decreases to $81 \mathrm{~V}$. The contribution of the splitter sheath on the total voltage is around $30 \mathrm{~V}$ in the following case. In case of two splitters, the presented times are not the same; indeed the 


\begin{tabular}{lllll}
\hline \hline Boundary conditions & Momentum & Enthalpy & Scalar Potential & Vector Potential \\
\hline Cathode & $\mathrm{v}=0 \mathrm{~m} / \mathrm{s}$ & Heat Transfer & Continuity & BiotSavart \\
Anode & $\mathrm{v}=0 \mathrm{~m} / \mathrm{s}$ & Heat Transfer & Continuity & BiotSavart \\
In & $/$ & $\mathrm{dT} / \mathrm{dn}=0$ & $\mathrm{I}=50 \mathrm{~A}$ & $/$ \\
Out & $\mathrm{v}=0 \mathrm{~m} / \mathrm{s}$ & $\mathrm{dT} / \mathrm{dn}=0$ & $0 \mathrm{~V}$ & $/$ \\
Vent In & $\mathrm{v}=0 \mathrm{~m} / \mathrm{s}$ & $300 \mathrm{~K}$ & $\mathrm{dV} / \mathrm{Dn}=0$ & BiotSavart \\
Vent Out & $\mathrm{P}=1 \mathrm{~atm}$ & Convection & $\mathrm{dV} / \mathrm{Dn}=0$ & BiotSavart \\
Walls & $\mathrm{v}=0 \mathrm{~m} / \mathrm{s}$ & $300 \mathrm{~K}$ & $\mathrm{dV} / \mathrm{Dn}=0$ & $/$ \\
Splitters & $\mathrm{v}=0 \mathrm{~m} / \mathrm{s}$ & Heat Transfer & Continuity & BiotSavart \\
\hline \hline
\end{tabular}

Table 1. Boundary conditions used in the simulation model.
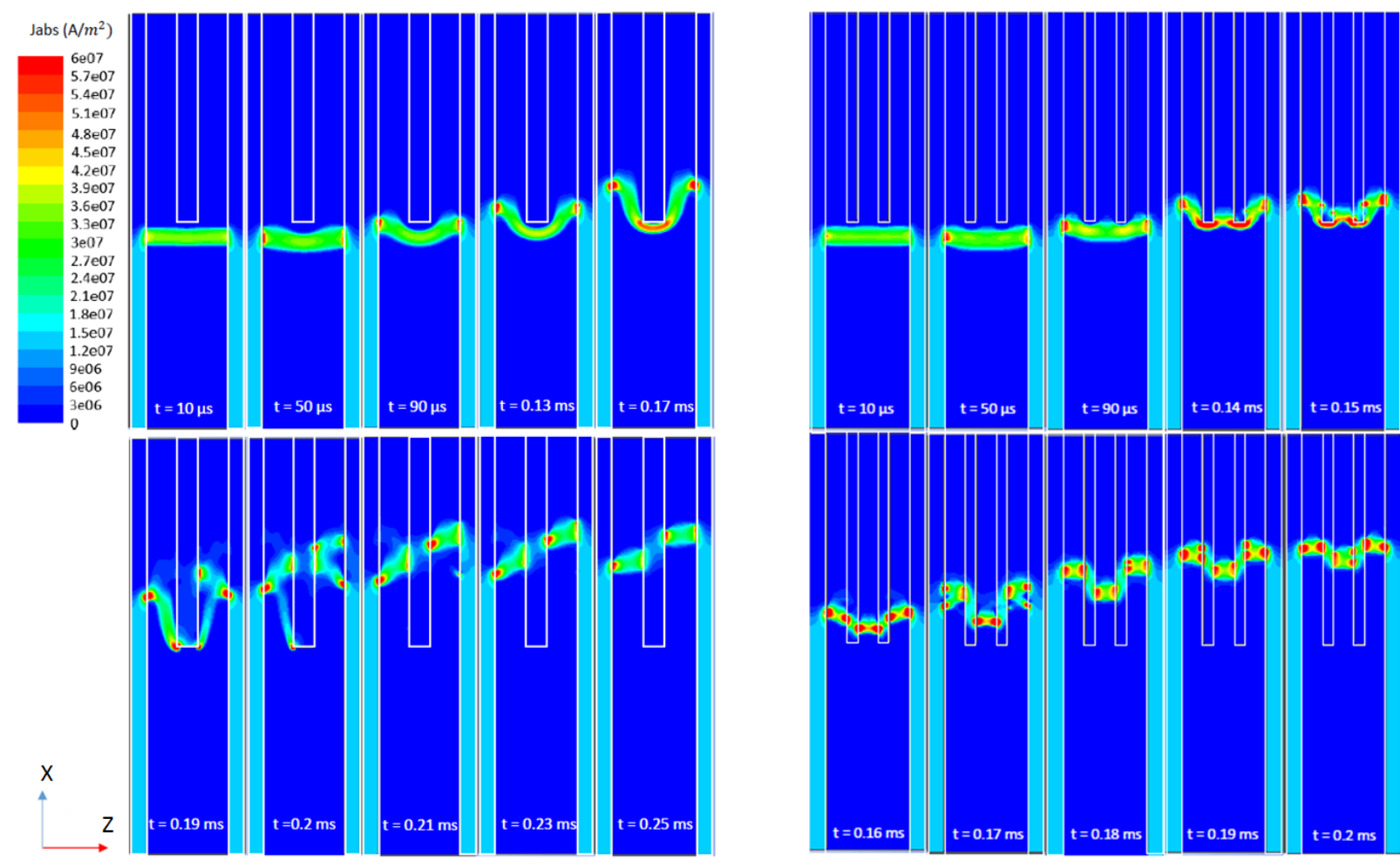

Figure 4. Simulated current densities using curve c for one (left) and two (right) splitters.

arc motion, the splitting process time and behavior are different. At $t=0.14 \mathrm{~ms}$, the arc column bends around the splitter plates, the arc voltage increases up to $76.6 \mathrm{~V}$. Compared to one splitter case, we can observe that at $t=0.15 \mathrm{~ms}$ the arc begins to jump on the splitters. From this time, two arc roots appear on the splitters plates, the current density through the splitter plates increases and the current path out of the splitters plates gradually fades. Compared with the case of one splitter, the arc voltage evolution is faster. At time $t=0.25 \mathrm{~ms}$ the difference on the total arc voltage between the two cases is only $4 \mathrm{~V}$. The comparison is difficult to make as the current paths and arc lengths are not the same. According to the results with one splitter an additional voltage $U=30 \mathrm{~V}$ between the two cases should exist. Nevertheless the arc positions are different, the medium temperature is different and the arc behavior not the same as shown by the voltage evolutions Figure 3 .

\section{Experimental results}

The dimensions of the experimental setup are the following: $126 \times 15 \times 20 \mathrm{~mm}(x y z)$ for the chamber, $126 \times 15 \times 1 \mathrm{~mm}(x y z)$ for the runners and $50 \times 15 \times 1 \mathrm{~mm}$ for the splitter plate. There is a wire located in front of the splitter to ignite the electrical arc. The experimental setup is composed by a generator which can product a prospective peak current up to $13 \mathrm{kA}$ with a maximum charging voltage of $600 \mathrm{~V}$ in AC, a high-speed camera (Photron SA5), a differential voltage probe and a Rogowski coil for the electrical measurements. Figure 5 presents the current evolution and the total voltages versus time for zero and one splitter. The first peak of voltage at $t=0.25 \mathrm{~ms}$ characterizes the arc ignition. After that, the arc moves toward to the upper side of the chamber. During the arc motion the voltage is nearly constant $60 \mathrm{~V}$ until $t=2 \mathrm{~ms}$. At this time the arc arrives in front of the splitter plate. In the case with 


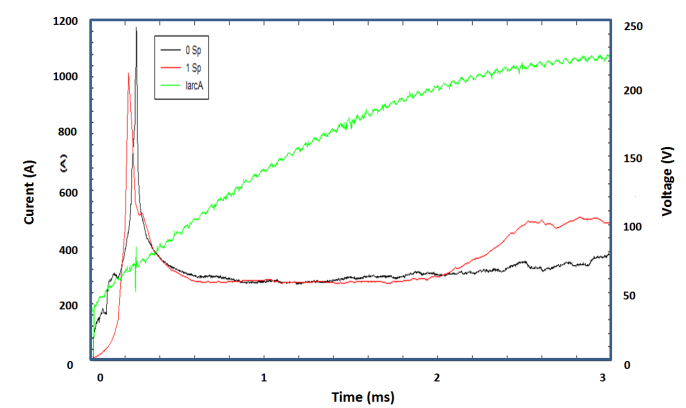

Figure 5. Experimental voltage drop and total current. Cases with one and without splitter in the chamber.

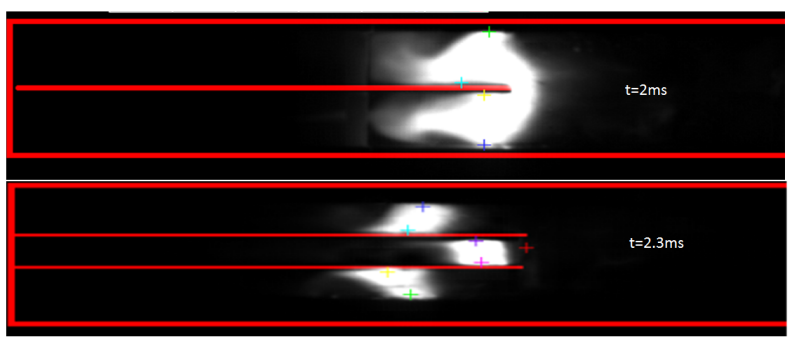

Figure 6. Example of arc behavior in front of one and two splitters plates obtained by high-speed camera.

one splitter, arc voltage increases by squeezing against the splitter, by touching the lower edge of the splitter until arc was totally on the splitter from $t=2 \mathrm{~ms}$ to $t=3 \mathrm{~ms}$. The voltage drop contribution dues to the splitter can be calculated by the difference between the case without splitter. The value is nearly $25 \mathrm{~V}$. This sheath contribution is in the same order of magnitude than the theoretical case, nevertheless we have seen between the two theoretical cases (one and two splitters) that the comparison is difficult due to the tortuous current paths or arc behavior in the plasma.

Finally we present Figure 6 two images obtained by the high speed camera to illustrate the arc behavior observed experimentally. In the upper picture the arc is bent in front of the splitter as in the down picture the arc is segmented by the presence of the two splitters plates.

\section{Conclusion}

A 3D model was developed and the effective electrical model of conductivity suggested by Lindmayer [2] introduced in the developments to characterize the additional voltage due to the splitter plate sheathes. The feasibility is demonstrated in cases of one and two splitters plates. Interesting results are obtained on the voltage evolutions. In the same time investigations are made to characterize the arc behavior in an experimental setup by high speed camera and electrical measurements. The theoretical and experimental configurations are at this time different nevertheless the same order of magnitude was observed with an additional drop voltage around $20-30 \mathrm{~V}$ for each splitter plate. The next step of our study will consist to use the same geometry for the model and the experience and to perform one parametric study on the number of splitters plates.

\section{References}

[1] J. Quéméneur, J. Lu, J.-J. Gonzalez, and P. Freton. Arc motion in low voltage circuit breaker (LVCB) experimental and theoretical approaches. Science and Technology, 8(2):35-45, 2018. doi:10.5923/j.scit.20180802.02.

[2] M. Lindmayer, E. Marzahn, A. Mutzke, T. Ruther, and M. Springstubbe. The process of arc splitting between metal plates in low voltage arc chutes. IEEE Trans. CPMT, 29(2), 2006. doi:10.1109/HOLM.2004.1353090.

[3] A. Mutzke, T. Ruther, M. Kurrat, M. Lindmayer, and E.-D. Wilkening. Modeling the arc splitting process in low-voltage arc chutes. In 53rd IEEE Holm Conf. on Electrical Contacts, Pittsburgh, 2007. doi:10.1109/HOLM. 2007.4318213.

[4] A. Mutzke, T. Ruther, M. Lindmayer, and M. Kurrat. Arc behavior in low-voltage arc chambers. European Physical Journal-applied Physics, 49, 2010. doi:10.1051/epjap/2010001.

[5] M. S. Benilov and A. Marotta. A model of the cathode region of atmospheric pressure arc. J. Phys. D: Appl. Phys., 28(9), 1995. doi:10.1088/0022-3727/28/9/015.

[6] F. Yang, M. Rong, and Y. Wu. Numerical analysis of arc characteristics of splitting process considering ferromagnetic plate in low-voltage arc chamber. IEEE Trans. Plasma Sci., 38(11), 2010. doi:10.1109/TPS. 2010.2070084.

[7] Z. Sun, M. Rong, and F. Yang. Numerical modeling of arc splitting process with ferromagnetic plate. IEEE Trans. on Plasma Sci., 36(4):1072-1073, 2008. doi:10.1109/TPS.2004.924559.

[8] M. Rong, F. Yang, Y. Wu, et al. Simulation of arc characteristics in miniature circuit breaker. IEEE Trans. on Plasma Sci., 38(9):2306-2311, 2010. doi:10.1109/TPS. 2010.2050703.

[9] F. Yang, M. Rong, Y. Wu, et al. Numerical simulation of the eddy current effects on the arc splitting process. Plasma Science and Technology, 14(11), 2012. doi:10.1088/1009-0630/14/11/05.

[10] F. Yang, M. Rong, Y. Wu, et al. Numerical analysis of the influence of splitter-plate erosion on an air arc in the quenching chamber of a low-voltage circuit breaker. J. Phys. D: Appl. Phys., 43(43), 2010. doi: $10.1088 / 0022-3727 / 43 / 43 / 434011$.

[11] J.-J. Lowke and M. Tanaka. Lte-diffusion approximation for arc calculations. J. Phys. D: Appl. Phys., 39(16), 2006. doi:10.1088/0022-3727/39/16/017.

[12] J. W. McBride and P. M. Weaver. Review of arcing phenomena in low voltage current limiting circuit breakers. IEE Proc. Sci. Meas. and Tech., 1(23), 2001. doi:10.1049/ip-smt: 20010185.

[13] J. W. McBride and D. Shin. A study of the motion of high current arcs in splitter plates using an arc imaging system. In International Conference on Electrical Contacts, Edinburg, 2016. 
[14] J. W. McBride, A. Balestrero, L. Ghezzi,

G. Tribulato, and K. J. Cross. Optical fiber imaging for high speed plasma motion diagnostics: Applied to low voltage circuit breakers. Rev. Sci. Instrum, 81(5), 2010. doi:10.1063/1.3428737.

[15] D. Shin, I. O. Golosnoy, and J. W. McBride. A study of arc modelling in low-voltage switching devices. In International Conference on the Computation of Electromagnetics Fields, 2017.
[16] D. Shin, J. W. McBride, and I. O. Golosnoy. Arc modeling to predict arc extinction in low-voltage switching devices. In IEEE Holm Conf. on Elec. Cont., 2018. doi : 10.1109/HOLM. 2018.8611712.

[17] A. Iturregi, B. Barbu, E. Torres, F. Berger, and I. Zamora. Electric arc in low-voltage circuit breakers: Experiments and simulation. IEEE Trans. Plasma Sci., 45(1):113-120, 2017. doi:10.1109/TPS.2016.2633400. 